121 INTERFERON STIMULATED LONG NONCODING RNA LNCRNA-CMPK2 FACILITATES NEUTROPHILS INTERFERON PRODUCTION BY TLR7/8 AGONIST IN SLE

${ }^{1} Z \mathrm{Xue}^{*},{ }^{1} \mathrm{Y}$ Tang, ${ }^{1} \mathrm{M}$ Dai, ${ }^{1} \mathrm{~S}$ Chen, ${ }^{1,2} \mathrm{~N}$ Shen. ${ }^{1}$ RenJi Hospital- School of MedicineShanghai JiaoTong University, Shanghai Institute of Rheumatology, Shanghai, China; ${ }^{2}$ Cincinnati Children's Hospital- Medical Centre, The Centre for Autoimmune Genomics and Aetiology CAGE, Cincinnati- Ohio, USA

\subsection{6/lupus-2017-000215.121}

Background and aims Neutrophils are important source of high interferon in SLE, we aimed to identify Long noncoding RNAs (LncRNAs) that can be strongly induced by interferon and simultaneously show different expression in neutrophils of SLE and healthy controls. We also investigated how this LncRNA modulate neutrophils interferon production.

Methods RNA-seq was performed in two series of samples, interferon stimulated neutrophils samples and SLE versus healthy controls neutrophils samples. LncRNA-CMPK2 was screened out by cross-reference the two RNA-seq results. Neutrophils interferon production was measured by $\mathrm{qPCR}$ and ISRE report gene assay after LncRNA-CMPK2 was knocked down using antisense oligos electrotransfection.

Results SLE neutrophils produced more interferon when stimulated by TLR7/8 agonist R848 as compared to healthy controls. Neutrophils enhanced interferon production capacity after interferon prime. LncRNA-CMPK2 was an interferon stimulated LncRNA in neutrophils and had an expression level correlated with SLE disease activity. Knock down LncRNACMPK2 attenuated neutrophils interferon production.

Conclusions Interferon can augment neutrophils interferon production capacity in regenerative feedback. LncRNA-CMPK2 was an important interferon stimulated LncRNA and can facilitate neutrophils interferon production in SLE. Accommodate the expression of LncRNA-CMPK2 could probably supply a new thread of thought to SLE treatment.

\section{DENDRITIC CELLS DISPLAY ABERRANT TOLL-LIKE RECEPTOR 7/9 RESPONSES IN SYSTEMIC LUPUS ERYTHEMATOSUS}

A.L.Y. Yim, S Yan, A Chan, VSF Chan, CS Lau. LKS Faculty of Medicine- The University of Hong Kong, Department of Medicine, Hong Kong, Hong Kong S.A.R

\subsection{6/lupus-2017-000215.122}

Background and aims Systemic lupus erythematosus (SLE) is a multifactorial autoimmune disease that causes multi-organ damages. Plasmacytoid dendritic cells (pDCs) are potent type I interferon (IFN) producers and myeloid dendritic cells (mDCs) are professional antigen presenting cells. Clinically, serum IFNalpha (IFNa) level correlates with disease severity and mDCs from patients also display activated phenotypes. These observations suggested that different DCs subsets may mediate SLE pathogenesis. Therefore, the aim of this study was to evaluate whether pDCs and mDCs possessed aberrant properties that might mediate SLE progression.

Methods The properties of DCs from the murine lupus model New Zealand Black/White F1 (BWF1) were evaluated using flow cytometry, ELISA and qPCR.
Results Splenic pDCs abundance was similar before and after disease onset. The induction of CD40, CD80 and MHC II on pDCs upon Toll-like receptor (TLR) 7 or TLR9 stimulation and the level of IFN-alpha produced by pDCs in symptomatic and pre-symptomatic mice was also comparable. In contrast, splenic mDCs expanded in symptomatic mice. These mDCs decreased CD80 and MHC II expression but their ability in stimulating allogenic $\mathrm{T}$ cell proliferation was similar to $\mathrm{mDCs}$ from pre-symptomatic mice. On the other hand, TLR7 and TLR9 expressions in BWF1 mDCs were higher than mDCs from age- and sex-matched parental NZW controls. The amount of IL-10 and CXCL13 produced by mDCs from symptomatic mice upon TLR7 or TLR9 stimulation was also higher than its pre-symptomatic counterparts.

Conclusions Myeloid DCs displayed heightened TLR7 and TLR9 responses in SLE. More work is needed to further dissect how mDCs promote SLE pathogenesis.

\section{MIR-127-3P AS A NOVEL REGULATOR OF TYPE I INTERFERON SIGNALLING PATHWAY IN SLE}

H Zhou* ${ }^{*}$ N Shen. Autoimmune Research Centre, Renji Hosipital, Shanghai, China

\subsection{6/lupus-2017-000215.123}

Background and aims Type 1 interferon(IFN) is a critical pathogenic factor in Systemic Lupus Erythematosus(SLE) and its associated nephritis, as elevated IFN inducible genes have been found in the kidney tissues and deficiency of IFN receptor protects lupus mouse model from developing nephritis. In this study, we want to find if there are miRNAs abnormal in the kidneys of lupus patients.

Methods miRNAs were quantified by RT-qPCR. Interferonstimulated response element(ISRE)-luciferase reporter assay and western blotting were used to investigate the function of candidate miRNAs. Genes that were affected by specific miRNA were identified by microarray. Antagomir(chemical modified miRNA inhibitors) was used to inhibit the function of candidate miRNA to validate its function. We administrated agomir (chemical modified miRNA mimics) into pristane induced pulmonary haemorrhage $(\mathrm{PH})$ mouse model to investigate the in vivo function of the candidate miRNA.

Results The expression of miR-127-3p decreased in kidney tissues from lupus nephritis patients and pristane induced lupus mice. miR-127-3p was found negatively regulating the type 1 IFN signalling by directly targeting JAK1 and knocking down of miR-127-3p enhanced type 1 IFN signalling. Overexpression of miR-127-3p prevented pristane induced lung haemorrhage.

Conclusions Our study shows miR-127-3p can inhibit IFN signalling and is reduced in kidneys of lupus nephritis patients indicating a new mechanism of overactivated IFN response in the kidney of SLE. In vivo inhibitory effects of miR-127-3p on IFN signalling suggest its therapeutic potential of treating lupus. Ongoing mouse model studies about the effects of miR$127-3 p$ on lupus nephritis will give us more insights into its therapeutic value. 